\title{
Enhancing acoustic cavitation using artificial crevice bubbles
}

\author{
Aaldert Zijlstra $^{\mathrm{a}}$, David Fernandez Rivas ${ }^{\mathrm{b}, *}$, Han J.G.E. Gardeniers ${ }^{\mathrm{b}}$, Michel Versluis ${ }^{\mathrm{a}}$, Detlef Lohse ${ }^{\mathrm{a}}$ \\ ${ }^{a}$ Physics of Fluids Group, MESA + Institute for Nanotechnology, University of Twente, P.O. Box 217, 7500 AE Enschede, The Netherlands \\ ${ }^{\mathrm{b}}$ Mesoscale Chemical Systems Group, MESA + Institute for Nanotechnology, University of Twente, 7500 AE Enschede, The Netherlands
}

\section{A R T I C L E I N F O}

\section{Article history:}

Received 27 January 2014

Received in revised form 29 September 2014

Accepted 1 October 2014

Available online 18 October 2014

\section{Keywords:}

Ultrasound

Cavitation

Nucleation

Bubble

High-speed imaging

\begin{abstract}
A B S T R A C T
We study the response of pre-defined cavitation nuclei driven continuously in the $\mathrm{kHz}$ regime $(80,100$ and $200 \mathrm{kHz}$ ). The nuclei consist of stabilized gaspockets in cylindrical pits of $30 \mu \mathrm{m}$ diameter etched in silicon or glass substrates. It is found that above an acoustic pressure threshold the dynamics of the liquid-gas meniscus switches from a stable drum-like vibration to expansion and deformation, frequently resulting in detachment of microbubbles. Just above this threshold small bubbles are continuously and intermittently ejected. At elevated input powers bubble detachment becomes more frequent and cavitation bubble clouds are formed and remain in the vicinity of the pit bubble. Surprisingly, the resulting loss of gas does not lead to deactivation of the pit which can be explained by a rectified gas diffusion process.
\end{abstract}

(c) 2014 Elsevier B.V. All rights reserved.

\section{Introduction}

Most applications based on acoustic cavitation, such as ultrasonic cleaners, emulsifiers or sonochemical reactors, rely on the spontaneous and seemingly random appearance of cavitation bubbles [1-3]. The subsequently generated cavitation state, meaning the bubble sizes, locations, dynamics and complex bubble-bubble interactions among others [4], are very difficult to describe and even more difficult to control [5]. This is mainly due to the inherent chaotic nature of cavitation and its complex dependence on the liquid and acoustic parameters. Manufacturers and researchers therefore have to resort to empirical methods to optimize the desired mechanical or chemical effects induced by acoustic cavitation bubbles [6-9]. The resultant of these effects is not only determined by the magnitude of the response of each individual bubble, but also by the total number of bubbles and their locations. The increase of cavitation activity would seem just a matter of increasing the acoustic amplitude, or input power. However, due to effects such as acoustic shielding and bubble-bubble interactions, this does not necessarily lead to an increase in efficiency [10-12]. There is also a frequency dependent lower acoustic power threshold for cavitation activity, usually referred to as the nucleation threshold [13]. Hence in the situations where lower acoustic amplitudes are required, e.g. to prevent heating or surface damage [14], it might not even be possible to generate or sustain a sufficiently active bubble population.

\footnotetext{
* Corresponding author.

E-mail address: d.fernandezrivas@utwente.nl (D. Fernandez Rivas).
}

Enhancing cavitation mediated effects can be achieved by actively regulating not only the acoustic pressure amplitude, but also the bubble population. Therefore, an ideal solution is to introduce microbubbles of resonant size into the liquid. Various studies have shown that the presence of ultrasound contrast agents microbubbles stabilized by an external shell - significantly enhances cavitation related effects $[15,16]$. Other methods such as bubble injection using gas flow through membranes [17], or flow focusing devices [18] could also be beneficial for increasing bubble populations in acoustic cavitation.

Here we present results of a different method to locally enhance acoustic cavitation activity in a continuous ultrasound pressure field. Our method consists of introducing microbubbles into the liquid which are entrapped and stabilized in micromachined and well defined cylindrical crevices, an image of which is shown in Fig. 1(b). These bubbles are essentially artificial versions of naturally occurring cavitation nuclei better known as "crevice bubbles" $[19,20]$. Bubble detachment from crevice bubbles occurs, not only in cavitation inception, but also in supersaturated liquids such as carbonated beverages [21,22] and in liquid pool boiling [23]. The use of artificial crevice bubbles was motivated by earlier studies of our group [24-27] where the response of the entrapped bubble to a single shock wave was investigated. Here we study the dynamics of stabilised cylindrical pit bubbles under continuous ultrasound irradiation. It will be shown that the presence of stabilised microbubbles significantly enhances local cavitation activity due to acoustically induced microbubble detachment from the pit bubble liquid-gas interface. 
A spherical bubble dissolves under ambient pressure and surface tension or rises to the surface due to buoyancy, while an entrapped bubble can be in a stable mechanical and diffusive equilibrium for an extended period of time. The presence of a crevice bubble nucleus basically constitutes a "weak-spot" in the liquid. Any change in the pressure or gas saturation conditions will cause a volumetric response of the bubble as it seeks to re-establish an equilibrium state. Nucleation is the event where the equilibrium cannot be established and the bubble expands out of the pit $[20,27]$. The three different but interrelated ways for this to occur are pressure reduction, increase in gas saturation or by a rise in temperature.

It is important to make a distinction between nucleation, i.e. the unstable growth of the bubble outside the pit, and a possible bubble detachment from the nucleus. Many authors use the term nucleation for the combined process, however the underlying mechanisms are different. Nucleation refers to the observable unstable growth of the liquid gas interface. Once nucleated, the bubble can become elongated and form a contracting neck until detachment takes place. This process which we refer to as pinchoff can occur due to buoyancy, liquid drag or acoustic pressure gradients. However, as will be shown, detachment of a bubble from the interface can also occur without bubble nucleation.

Bubble detachment following nucleation is a well studied effect in nucleate boiling [28] and particularly in supersaturated liquids [22] but surprisingly not in acoustic cavitation. Even though bubble detachment from entrapped surface bubbles is normally assumed to occur in acoustic cavitation, to our knowledge, there exist only a few studies in which this is actually reported. The first experiment reported consisted in holes of $200 \mu \mathrm{m}$ diameter drilled in a sonotrode tip, in order to enhance cavitation effects at reduced acoustic amplitudes [29]. The growth of bubbles, surface instabilities and bubbles leaving the holes were observed. Erosion studies on brass surfaces from bubbles nucleated from a bubble stabilised on a pit of $1 \mathrm{~mm}$ diameter and depth, drilled on the sonotrode surface at $20 \mathrm{kHz}$, have also been reported. In degassed water and in the absence of the drilled pit, erosion effects were not observed [30].

Here we are interested in the response of artificial pit bubbles to a continuous acoustic field, and how its response can contribute to the enhancement of cavitation activity. In the following, the process of bubble nucleation and detachment by pinch-off in acoustic cavitation is investigated in more detail using high-speed imaging methods.

\section{Materials and methods}

\subsection{Machining of pits}

In order to obtain pit bubbles which are comparable in size with naturally occurring crevice bubbles, but large enough to allow visual observations, we chose to create cylindrical pits of $30 \mu \mathrm{m}$ in diameter. The pits with depths of $\sim 10 \mu \mathrm{m}$ and $6 \mu \mathrm{m}$ were etched both in polished silicon wafers as well as in fused silica glass, see Refs. $[10,12]$ for more details. We found by optical inspection that a stable liquid-gas micromeniscus was always present after immersion of the substrate even though no extra measures were taken to increase the hydrophobicity of the pit as was done in the past $[24,25]$.

\subsection{The acoustic field}

In this study, three setups were used. The silicon substrates were placed on the bottom of one of two small containers providing stability, liquid containment and convenient optical access. The first type of container was a cubic cuvette (Hellma Analytics), made of optical quality quartz glass with inner dimensions $1 \times 1 \times 1 \mathrm{~cm}$. The second type was cylindrical with an inner diameter of $1.5 \mathrm{~cm}$ and a depth of $3 \mathrm{~mm}$. The acoustic field was generated by means of a piezoelectric element (Ferroperm PZ27, $6 \mathrm{~mm}$ thickness and diameter of $2.5 \mathrm{~cm}$ ) glued to the containers (see Fig. 1). The maximum response of the piezo and cuvette combination when filled with water was found to occur at frequencies close to $80 \mathrm{kHz}$ and $200 \mathrm{kHz}$ corresponding to radial and thickness vibration modes.

In order to take full advantage of the transparency of the glass substrates with pits, a system described in the literature was used [31]. Here, the glass substrates together with four piezo elements were glued to a glass microscope slide. Upon adding demineralized water (left standing for one day to ensure full saturation) onto the substrates, a pocket of air was entrapped within the cylindrical pits. The presence of the liquid-gas interface was verified using dark-field illumination microscopy.

The piezoelectric elements of the cuvette systems were actuated using a continuous sinusoidal voltage generated by a function generator (model WW1072, Tabor Electronics Ltd.) which was amplified using a wide band amplifier (Krohn-Hite, model 7602). The glass system was actuated by an arbitrary wave generator Agilent 33522A and amplified with an AG series amplifier T\&C Power Conversion. The input voltage and current signal were measured using an oscilloscope (Tektronix, DPO 4043) and a current probe (Tektronix, CTA-2). The small liquid volume and the close proximity to the piezo element complicated the pressure measurements, therefore the input electrical power was taken as a relative measure of power. The acoustic power cannot be directly quantified for these values.

The applied acoustic field is continuous, therefore a standing wave is formed in the container depending on the shape and dimensions of the liquid volume. It was found that the largest response of the pit bubble occurred for liquid heights equal to

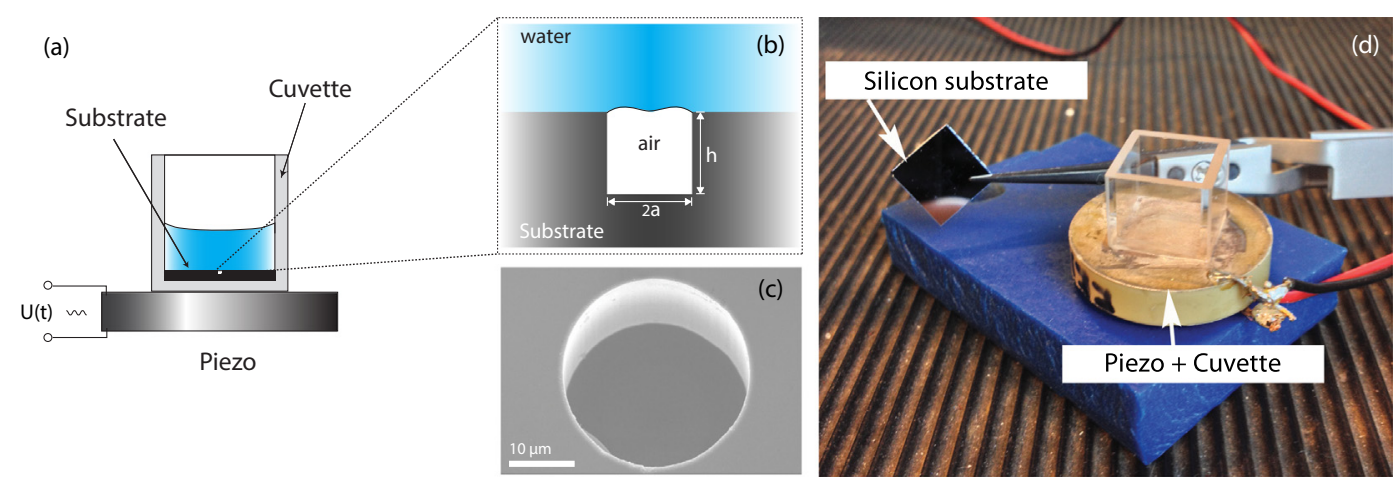

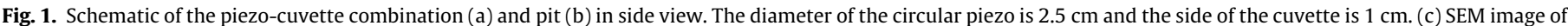

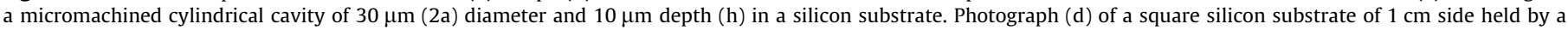
tweezer, and piezo glued to the quartz glass cuvette. 
one-quarter or three-quarters of the acoustic wavelength. This situation corresponds to a pressure antinode situated at the substrate and a pressure node at the free water-air interface. For the two cuvette systems heights equal to $\lambda / 4=4.7 \mathrm{~mm}$ for $80 \mathrm{kHz}$ and $3 \lambda / 4=5.6 \mathrm{~mm}$ for $200 \mathrm{kHz}$ were set by manual filling of the cuvette. For the transparent glass substrate system, a frequency of $99 \mathrm{kHz}$ was used corresponding to an effective quarter wavelength liquid height of $3.8 \mathrm{~mm}$.

\subsection{Imaging methods}

The timescale of acoustic cavitation events is dictated by the period of the driving acoustic field (order $10 \mu \mathrm{s}$ ). Hence, in order to resolve the dynamics of ultrasonically driven (pit) bubbles and resulting bubble cloud, high-speed imaging techniques are required. Here we use two types of high-speed cameras: the Photron, model SA1.1 with a framerate up to $500 \mathrm{kfps}$ and the Brandaris 128 capable of frame rates up to $25 \mathrm{Mfps}[32,33]$.

High-speed cameras allow for the visualization of extremely fast events, however the limited spatial resolution of the produced images limits image analysis. Therefore an additional ultrashort single flash imaging method was applied which enabled recording of high resolution images without motion induced blur. The setup used for this particular imaging method is depicted in Fig. 2. The illumination source consists of a pulsed laser (Nd:YAG, Litron, model Nano S 65-15 PIV, wavelength $532 \mathrm{~nm}$ ) with a pulse duration of $7 \mathrm{~ns}$ (full width at half maximum) and a maximum pulse energy of $65 \mathrm{~mJ}$. The laser pulse is directed into a quartz cuvette (Hellma Quartz Suprasil, dimensions $10 \times 10 \times 40 \mathrm{~mm}$ ) containing fluorescent laser dye dissolved to saturation in ethanol (LDS 698, Exciton Inc.). The geometry of the cuvette combined with the concentrated dye solution results in high-intensity fluorescence emerging at an angle of $90^{\circ}$ with the exciting laser beam [34]. More details of this method are described elsewhere [11,35]. The fluorescent illumination can be used in back-lighting configuration for side view or in reflective mode (top view, Fig. 2) enabled by the reflectivity of the silicon substrate. For this purpose the light pulse is relayed using a glass fiber and coupled into the microscope (Olympus). Image magnification was obtained with a long working distance objectives (LMPLFLN $10 \times$, Olympus, WD $21 \mathrm{~mm} /$ $\mathrm{NA}=0.25$ or the SMPLFLN $20 \times$, Olympus, WD $25 \mathrm{~mm} / \mathrm{NA}=0.25$ ). The illumination flash was synchronized with the single frame camera (Lumenera LM165, pixel size of $6.45 \times 6.45 \mu \mathrm{m}$, resolution

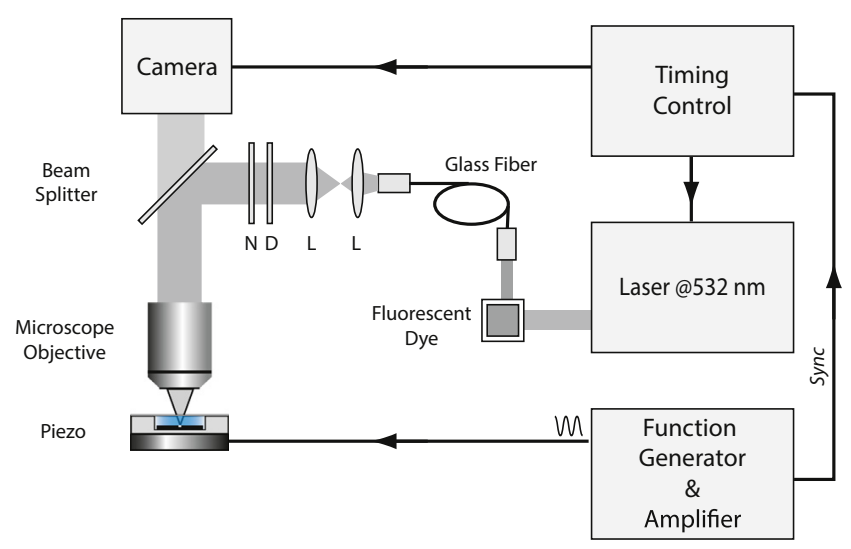

Fig. 2. Schematic overview of the setup used for single flash imaging of pit bubble pinch-off and cavitation in top-view. After exiting the fiber the beam is diffused (D) and filtered from any remaining laser light using a notch-filter (N) (Semrock, NF01$532 U-25)$. Illumination of the full field of view is achieved by adjusting the angle of divergence of the beam using two positive lenses (L).
$1392 \times 1040$ ) using a pulse delay controller (Berkeley Nucleonics, model 575). The delay controller was synchronized with the acoustic driving signal.

\section{Results}

\subsection{Cubic cuvette}

Here the cuvette system was used in order to enable side-view imaging using the microscope and high speed camera (Photron SA1.1) combination. We studied the pit bubble driven by different frequencies in a standing acoustic field.

\subsubsection{Bubble pinch-off at $80 \mathrm{kHz}$}

For small input powers, up to $\sim 50 \mathrm{~mW}$, there was no visible response of the pit bubble. For higher input powers, streams of microbubbles of various sizes were observed, which were ejected intermittently from the pit bubble. In the recordings of repeated experiments two different types of pinch-off phenomena were observed. For the first type the liquid-gas interface did not extend visibly outside the pit while bursts of microbubbles were expelled upwards, originating from different locations in the pit (Fig. 3(a)). In the second type of pinch-off (Fig. 3(b)) the pit bubble expands hemispherically outside the pit during the low pressure phase of the acoustic field and retracts into the pit when the pressure increases.

A sequence of frames of a high-speed movie of a bubble pinchoff event in the second regime is shown in Fig. 4. Here a microbubble of approximately $5 \mu \mathrm{m}$ in diameter is detached during the collapse phase of the micromeniscus between $20 \mu \mathrm{s}$ and $22 \mu \mathrm{s}$. The interface of the micromeniscus changes from its initial hemispherical shape to a bell-shape during the collapse phase. Occasionally the radial flow focusing is sufficient to overtake the top part of the interface thereby separating it from the rest of the meniscus. In all of the observed pinch-off events of this type, the bubble separates from the meniscus during the contraction phase, corresponding to the high pressure phase of the acoustic cycle. The resulting stream of microbubbles is visible in Fig. 3(b). Because of the axial symmetric character of the pinch-off also the trajectories of the bubbles are mainly along the vertical axis. This observation will be discussed later in Section 4.

Estimates of the bubble sizes and pinch-off rates were obtained using image processing techniques implemented in Matlab
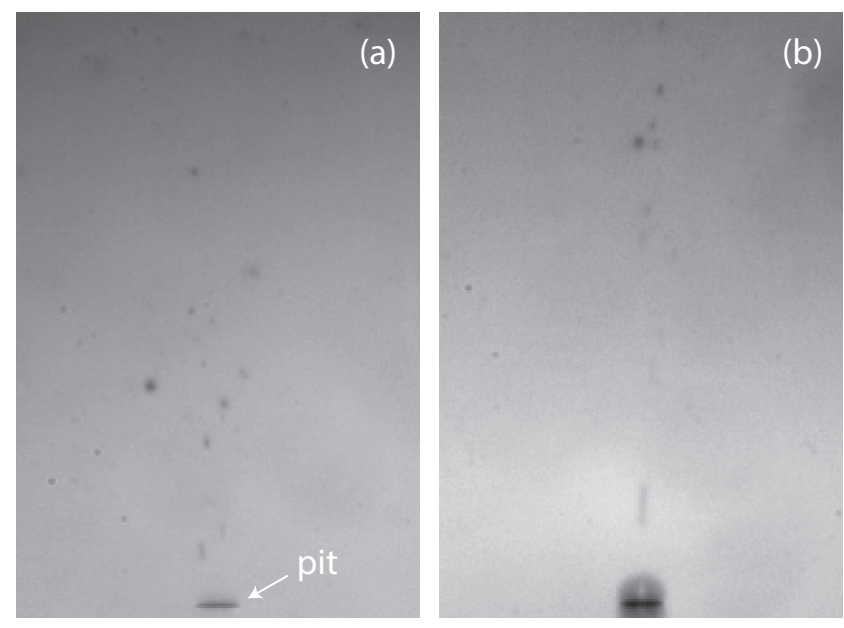

Fig. 3. Selected frames of a pit bubble expelling microbubbles. The left image (a) shows irregular bubble pinch-off where microbubbles move upward in various angles with the vertical axis. The right image (b) shows a regime where the pit bubble extends outside the pit perimeter and the pinched off bubbles move upwards along the vertical axis. Supplementary Video. 

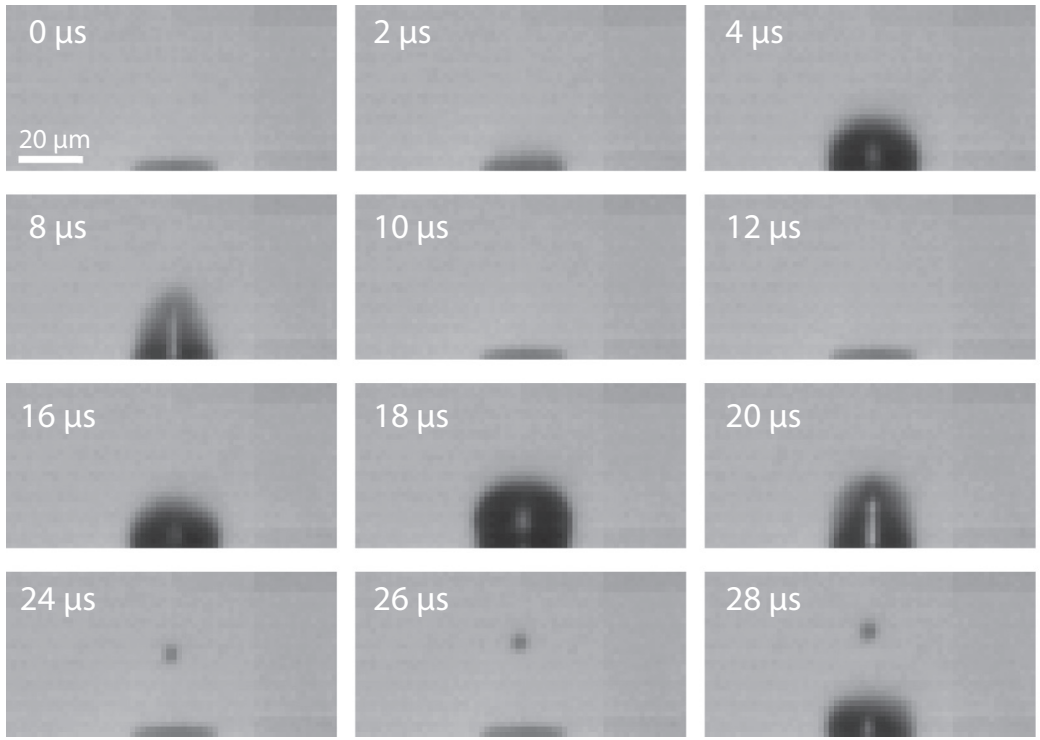
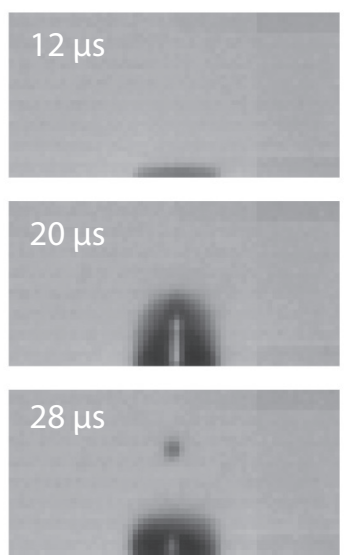

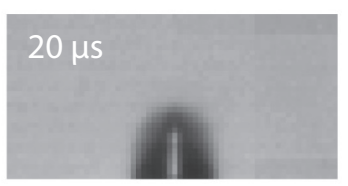

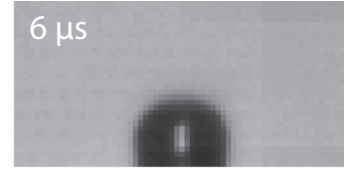
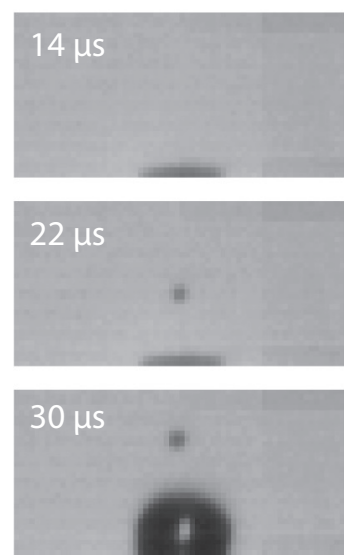

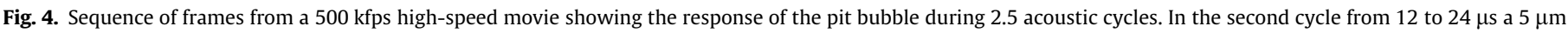
diameter microbubble is pinched off.

$\mathrm{R} 2009 \mathrm{~b}^{\circledR}$. Due to motion blur in the high speed videos only the trajectories of bubbles emerging some $100 \mu \mathrm{m}$ above the pit could be resolved. Two obtained time traces are shown in Fig. 5, one for the irregular type pinch-off (a) and one for the regular type pinch-off (b). It reveals that the bubble pinch-off processes are quite erratic, both in pinch-off rate, as well as the ejected bubble sizes. At some instances (e.g. near $60 \mathrm{~ms}$ in Fig. 5(a)) bursts of multiple bubbles with radii less than $5 \mu \mathrm{m}$ are expelled, but it can take several milliseconds before the next pinch-off event. The average rate of bubble pinch-off for these traces was $2 \mathrm{kHz}$ or every 40 acoustic cycles. The bubble size distributions shown in the insets in (a) and (b) show that the majority of the ejected bubbles had radii less than $7 \mu \mathrm{m}$, whereas the larger bubbles form during bubble coalescence. The irregular type pinch-off process results in bubbles with radii near $2 \mu \mathrm{m}$ with a broader size distribution. Bubble coalescence was more prevalent in the first type, forming larger bubbles compared to that of the second type as reflected in the bubble distributions. Bubbles smaller than $\mu \mathrm{m}$ in radius were observed but a precise quantitative determination of their radius was not possible due to limited image resolution.

For the regular type of pinch-off it was found that the velocity of the bubbles immediately after pinch-off was typically as high as $2 \mathrm{~m} / \mathrm{s}$ and slowing down to about $50 \mathrm{~mm} / \mathrm{s}$ at $200 \mu \mathrm{m}$ above the substrate. For the irregular type pinch-off the velocities were found to be an order of magnitude lower. The larger number of bubble coalescence events observed in this regime compared to the regular type can be related to these lower velocities.

\subsubsection{Driving at $200 \mathrm{kHz}$}

Here the cuvette setup is used again but now imaged in top view with the Brandaris 128 high-speed camera. The driving frequency is approximately twice the linear resonance frequency $(98 \mathrm{kHz})$ of the pit and lies below the first higher harmonic of $243 \mathrm{kHz}$ [36]. Similar to previous observations, there is a threshold for the pressure above which bubble pinch-off occurs corresponding to an input power of $50 \mathrm{~mW}$. Interestingly, in the regime below this threshold the liquid-gas interface exhibits alternating dark and bright rings which appear to move radially outward periodically with the driving frequency (see Fig. 6(a)).

A sequence of frames taken from a high-speed movie is shown in Fig. 6(b) with driving pressure slightly above the threshold
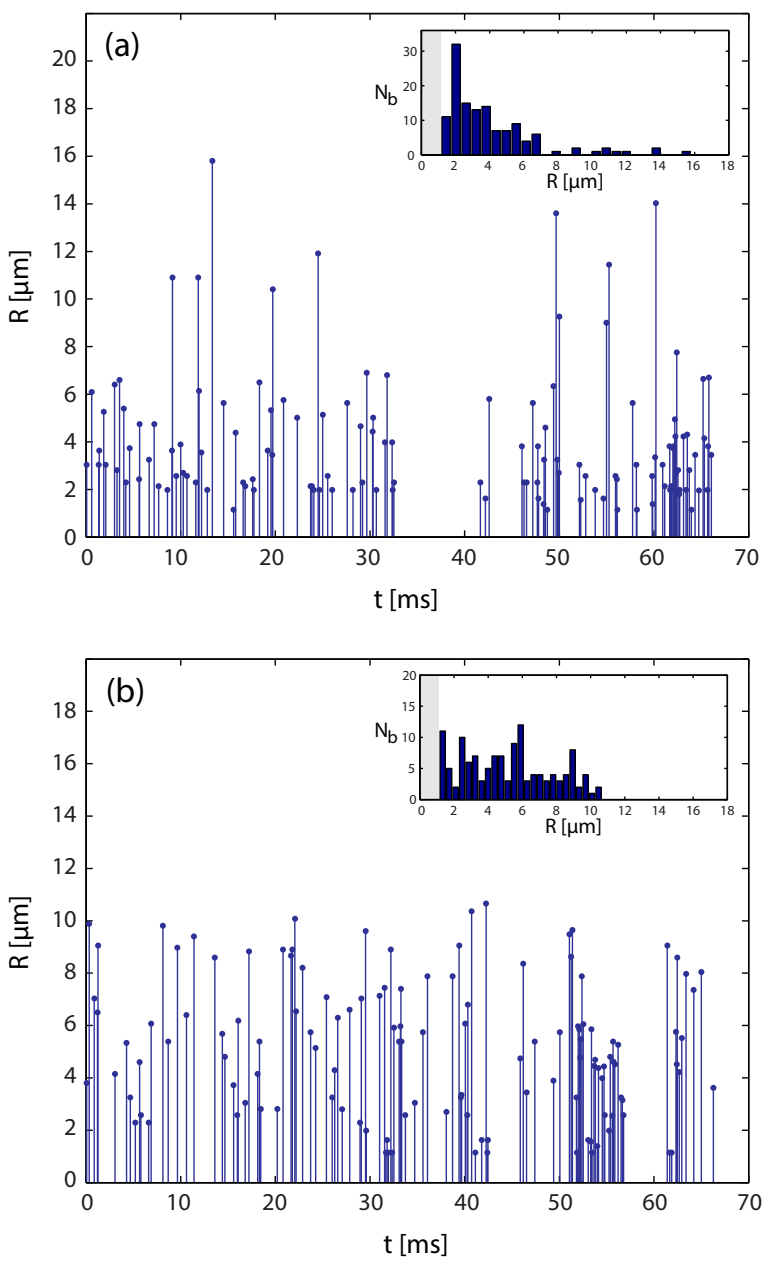

Fig. 5. Ejected bubble events marked by bubble radius in time for the irregular type pinch-off (a) and regular type pinch-off (b). The insets show the histograms with $N_{b}$ the number of bubbles. The data was obtained from $15 \mathrm{kfps}$ high-speed movie recordings. The image resolution did not allow bubble sizing for radii smaller than $1.3 \mu \mathrm{m}$ (indicated by the gray areas in the distributions). 
( $50 \mathrm{~mW}$ ). Here the rings are no longer visible and occasionally bubbles are pinched off which in turn respond to the acoustic field by expansion and collapse. In this regime the bubbles that are pinched-off generally are drawn back into the pit bubble within at most 3 acoustic cycles due to mutual acoustic forces. In contrast to the $80 \mathrm{kHz}$ driven pit bubble, the ejected microbubbles do not move upward but remain in close proximity of the substrate. From many similar recordings in this regime it was observed that once a bubble is detached from the pit bubble, a subsequent pinch-off event will not occur as long as the bubble is not recaptured. As shown in Fig. 6(c), increasing the input power enhances the deformation of the interface and bubbles are continuously pinched-off from the liquid gas interface. Also the number of free bubbles oscillating just outside the pit perimeter increases as well as their
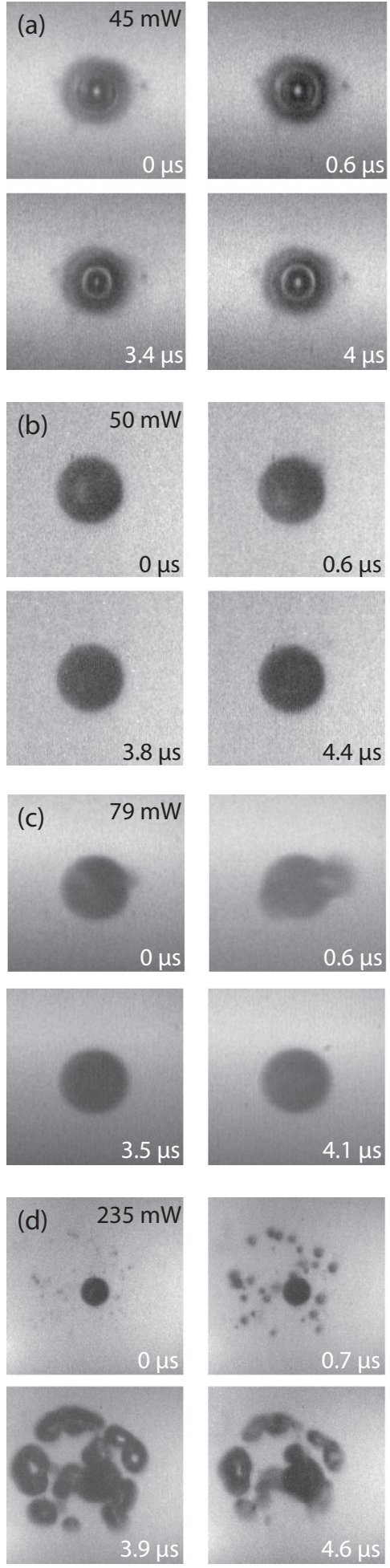
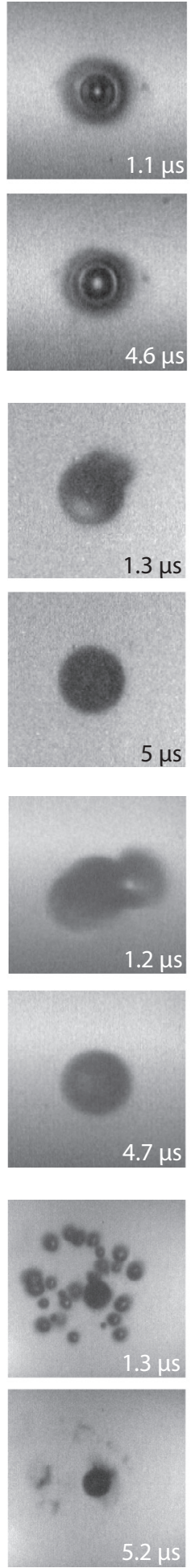
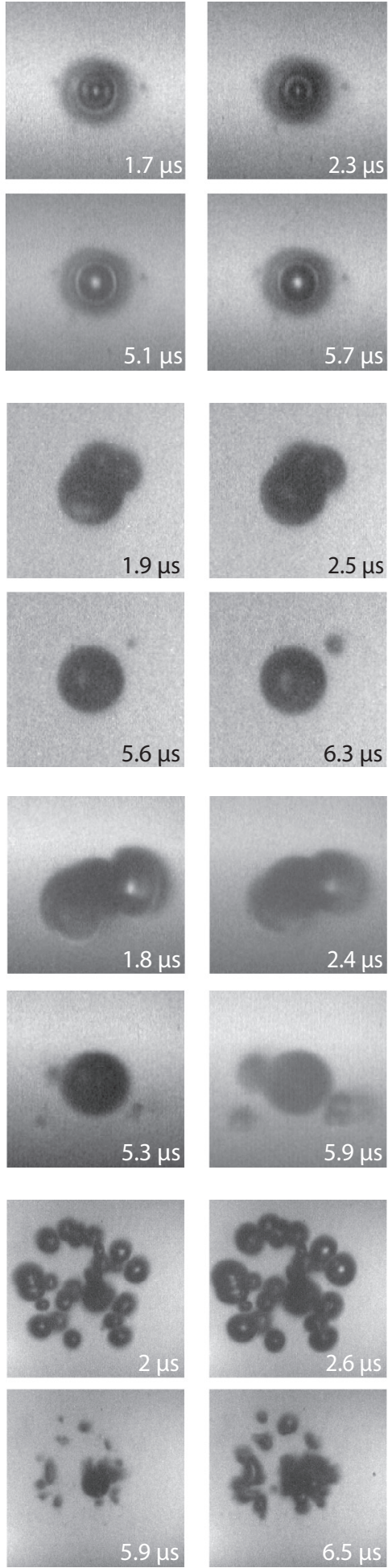
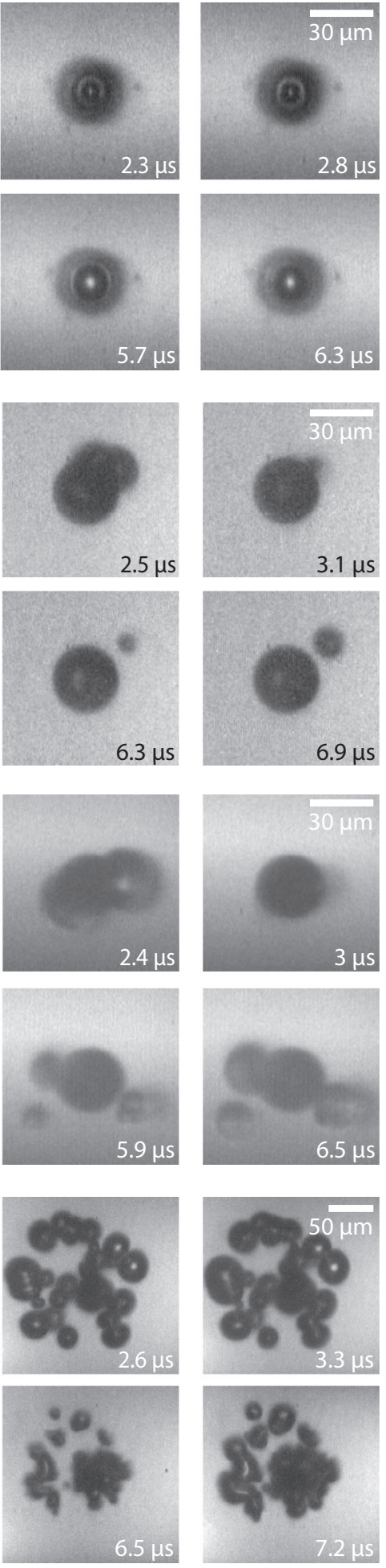

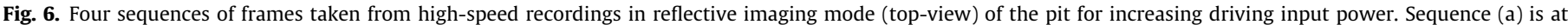

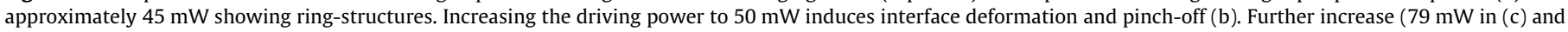
$235 \mathrm{~mW}$ in (d)) both increases the bubble number and maximum expansion. Note the different scale in (d). Supplementary Video. 
maximum expansion size. The bubbles still remain close to the pit bubble and are observed to oscillate up to 3 or 4 cycles before breaking-up or coalescing with the pit bubble or other bubbles.

The same trend is observed for larger driving input powers; more bubbles are pinched-off expanding to larger volumes with dimensions exceeding the diameter of the pit. As illustrated in Fig. 6(d) a cavitation bubble cloud is formed which is centered on the pit. Here both bubble deformation, coalescence and breakup are more pronounced compared to lower driving conditions (Fig. 6(b) and (c)). Due to these effects, the bubble population changes with every acoustic cycle. The response of an individual bubble in the cloud can therefore only be distinguished and followed in time during part of the expansion phase of the cycle (e.g. from $0.7 \mu$ s to $3.3 \mu \mathrm{s}$ ).

The increase in the number of bubbles with increasing driving input power is shown in the graph of Fig. 7. The estimates for the number of bubbles were obtained from high-speed recordings at different input powers. The number of bubbles varies considerably with each acoustic cycle (e.g. compare Fig. $6(\mathrm{~d})$ at $t=0.7 \mu \mathrm{s}$ and $t=6.5 \mu \mathrm{s}$ ) resulting in a large variance. Exact bubble counts were difficult to obtain due to the limited resolution of the optical system and due to the image processing detection limit when the pinched off bubbles were located close to the pit bubble or in contact with other bubbles.

The growth of the bubble cloud with increasing power is illustrated in Fig. 8. The frames in (a) show the scattered light from the bubbles in dark-field illumination mode giving an indication of the extent of the cloud. The corresponding frames in (b) obtained using iLIF imaging reveal the individual bubbles. From similar images the size of the bubble cloud was measured (Fig. 8(c)). Similar to what was observed at $80 \mathrm{kHz}$, the pit is not deactivated by loss of gas due to pinch-off processes. It was found that the cavitation cloud can remain stable for at least an hour, only to disappear when the acoustic field became disrupted due to evaporation of the liquid from the cuvette.

\subsection{Glass microscope slide}

The setup with the glass substrates enabled a better top-view imaging of the dynamics of the liquid-gas interface in the pit than the silicon substrates. A typical image sequence of the response of the interface to an $99.5 \mathrm{kHz}$ standing acoustic field obtained with this setup with voltage amplitude value $400 \mathrm{mV}$ is shown in Fig. 9. Further details on the characteristics and operation of this setup are reported in the literature $[31,37]$.

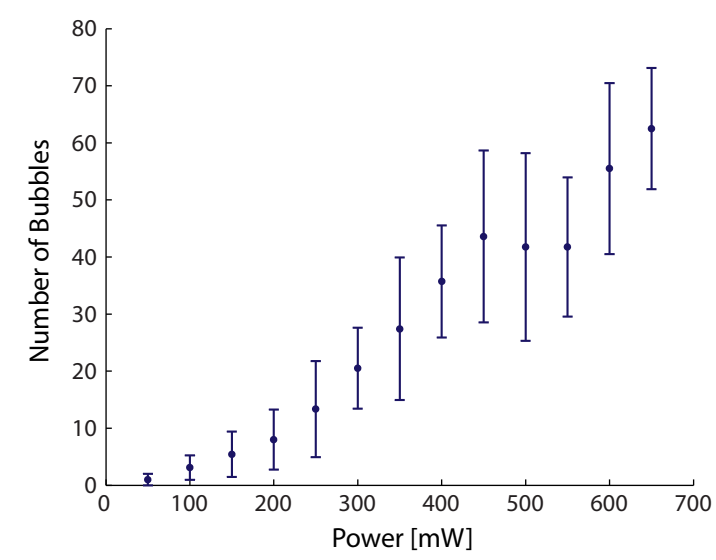

Fig. 7. Average number of bubbles during one acoustic cycle for different input powers at $200 \mathrm{kHz}$.

\subsubsection{Driving at $99.5 \mathrm{kHz}$, glass substrates}

Following the initiation of the acoustic field at $t=0$ the interface sequentially expands outward and back inward in a drum membrane-like fashion corresponding to the visual changes in brightness (first row). The oscillatory motion was occasionally interrupted as in the 5th frame. Here, during the inward motion the center of the interface comes into contact with the bottom of the pit which is shallower than those used in the silicon substrates. It appears that due to this contact the now toroidal interface becomes destabilized and detaches from the top right rim of the pit $(t=33.3 \mu \mathrm{s})$. In the following cycles the contact line of the gas volume retracts further until a circular shape is attained $(t=266.7 \mu \mathrm{s})$. Coinciding with this deformation a continuous stream of small bubbles is ejected from the bubble interface along the new axis of symmetry formed by the pit and the bubble (indicated by the white arrow at $t=277.8 \mu \mathrm{s}$ ). The bubbles are estimated to have diameters between 1 and $2 \mu \mathrm{m}$ and are ejected once every two acoustic cycles. A clear pinch-off event could not be distinguished from these recordings, however similar to Fig. 4, the detached bubbles emerge during the contraction phase of the liquid-gas interface.

Once the bubble attains its spherical shape it remains attached for an extended period of time to the lower left corner of the pit while oscillating with a maximum diameter (in lateral dimensions) of $20 \mu \mathrm{m}$ (see e.g. $t=288.9 \mu \mathrm{s}$ ). During the contraction phase, the bubble adapted an oval or bean-like shape. Occasionally, the bubble contraction occurred faster than the translation of the liquidgas contact line over the substrate resulting in semicircular bubble extensions (see e.g. $t=272.2 \mu \mathrm{s}$ or $t=2494 \mu \mathrm{s}$ ). At times, this lagging of the interface even resulted in a dual pinch-off from the edges of the bubble $(t=2506 \mu \mathrm{s})$. Another phenomena occurred when larger bubbles were formed either by pinch-off or coalescence $(t=9911 \mu \mathrm{s})$. These bubbles remained in proximity of the larger bubble, presumably due to secondary Bjerkness forces.

\subsection{Interaction of cavitation clouds and surface damage}

In Section 3.1.2 it was shown that in the presence of a single pit bubble, cavitation activity is initiated and sustained which would not occur without a pit under similar conditions. A natural solution to augment this activity is to introduce additional pits.

Indeed, as shown in the images in Fig. 10 another cavitation bubble cloud is generated from a second pit. Here the two pits (30 $\mu \mathrm{m}$ diameter) are $1000 \mu \mathrm{m}$ apart. At low driving power the individual clouds appear similar to the single pit system described in the previous section. At elevated powers $(648 \mathrm{~mW})$ the center of mass of the clouds is translated towards the neighboring pit through mutual attraction (Fig. 10(iv)). Increasing the power further, the clouds merge at the midpoint (Fig. 10(v) and (vi)). The transition between the two states occurs just above $648 \mathrm{~mW}$ driving power (iii) and is quite abrupt, meaning that the intermediate states between (iv) and (v) are unstable and very sensitive to pressure [4]. At higher power the cloud broadens, similar to the case of a single pit. Likewise, further increase in power does not produce a noticeable expansion of the cavitation cloud. The merging of the clouds results in a peculiar ribbon-like structure with a wider section in the middle measuring $\sim 200 \mu \mathrm{m}$ in diameter.

In order to illustrate the dynamics of the bubbles in the clouds a sequence of frames from a high-speed recording (Brandaris, 1.48 Mfps Supplementary Video) is shown in Fig. 11. The pits are located in the lower-right and upper-left corners. The first frame shows the bubble cloud with the bubbles at maximum expansion (similar to the frames (v) and (vi) in Fig. 10(b)). During the following cycles ( $5 \mu$ s duration) the majority of the bubbles in the cloud collapse and expand again in phase. A few considerably larger bubbles can be observed which, due to their size, exhibit a phase 

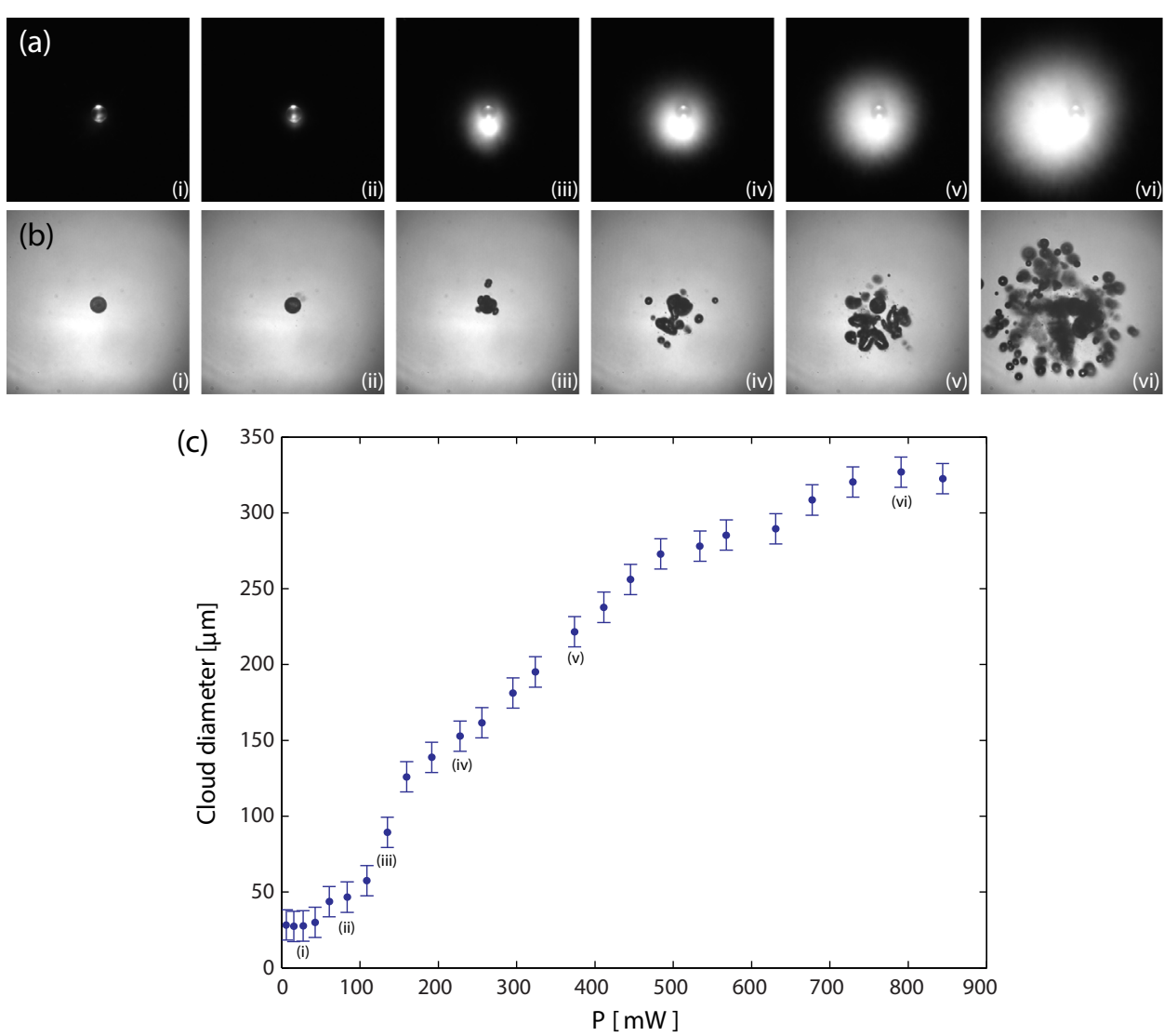

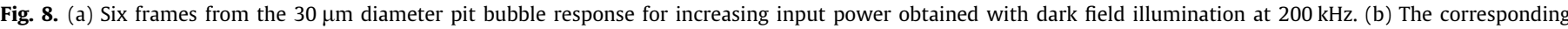

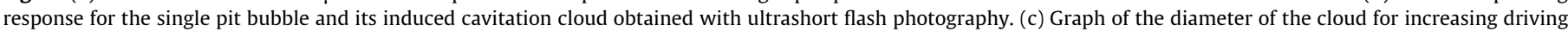
power. The frames (i)-(vi) in (a) and (b) are indicated in the graph.

difference with respect to the response of the other bubbles. For example the large bubble close to the lower-right corner continues its collapse until about $4 \mu$ s (7th frame) while the other bubbles have already started expansion. Similar to the single pit bubble cloud, the bubbles display coalescence, break-up and deformation, in particular the larger bubbles, due to the proximity to the substrate (attractive secondary Bjerknes forces as described elsewhere [4]). It is therefore difficult to track individual bubbles in these images. However the recordings do reveal that, on average, bubbles translate after pinch-off from both pits towards the center of the cloud. In this region a mist of small microbubbles can be observed during the high pressure phase of the acoustic cycle (e.g. at $2 \mu \mathrm{s}$ and $12.2 \mu \mathrm{s}$ ).

These small bubbles can result from increased bubble fragmentation taking place in this region combined with dissolution. Also in this case, the remarkable stability of the cloud, which was studied for at least one hour, suggests that the process is strongly dependent on diffusive transport of dissolved gas. In addition, the recordings shown in Figs. 8 and 10 demonstrate the resilience of the pit bubbles to dissolve. Evidently, even the strong pressure fluctuations associated with multiple bubble expansions and collapses are insufficient to destabilize the crevice bubble. A more detailed study of these type of bubble phenomena relevant for sonochemical activation is given elsewhere [11].

After several hours of US exposure a pattern of surface damage appeared on the substrate, which was consistent with the region of cavitation activity. The shock waves originating from the collapse of bubble clusters are also associated with the generation of surface damage. Further details on the damage of different crystallographic silicon substrates and practical uses for cleaning that these type of cavitation conditions can provide are given elsewhere $[38,39]$.

\section{Discussion}

Jetting has been defined in certain cavitation experiments as the inertia-driven passage of liquid flow through a gas bubble in the proximity of another bubble, or a wall. These bubbles collapse asymmetrically which can lead to physical changes in nearby cells, cleaning of substrates and erosion of surfaces [38-40]. A specific type of capillary break-up is also termed "jetting", but in this case refers to the ejection of small bubbles as a result of flow focusing of a gas in a liquid $[18,41,42]$.

For the experiments at $80 \mathrm{kHz}$ (Section 3.1.1) we could capture this second type of jetting, where the gas-liquid interface was perturbed by the strong oscillations driven by the piezo actuation. From the observations at all frequencies we speculate that the bubble pinch-off occur by a "folding" of the liquid-gas interface resulting from large amplitude capillary waves. These closed capillary waves resemble what is known as "Crapper wave" solutions [43]. The regime shown in Fig. 3(a) where bubbles are pinched-off from different locations on the interface then correspond to a shorter capillary wavelengths compared to the regime shown in Figs. 3(b) and 4. Another explanation is that the liquid-gas interface becomes deformed in such a way that the cylindrical symmetry of the pit bubble is broken, and the character and locations of pinch-off changes, as will be further discussed (see Fig. 9).

For the particular case of pinch-off at $80 \mathrm{kHz}$ driving (Section 3.1.1), all of the observed ejected bubbles, have radii much smaller than the linear resonance radius of $38 \mu \mathrm{m}$ [1], as expected. In comparison, the resonance frequencies for the first and second surface mode for this pit are $139 \mathrm{kHz}$ and $276 \mathrm{kHz}$ respectively, based on linear potential flow analysis [36]. This explains the larger amplitude response of the pit bubble compared to that of the ejected 

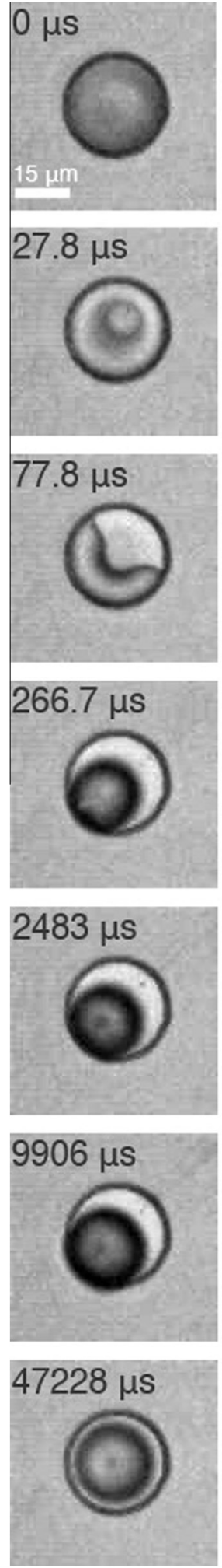
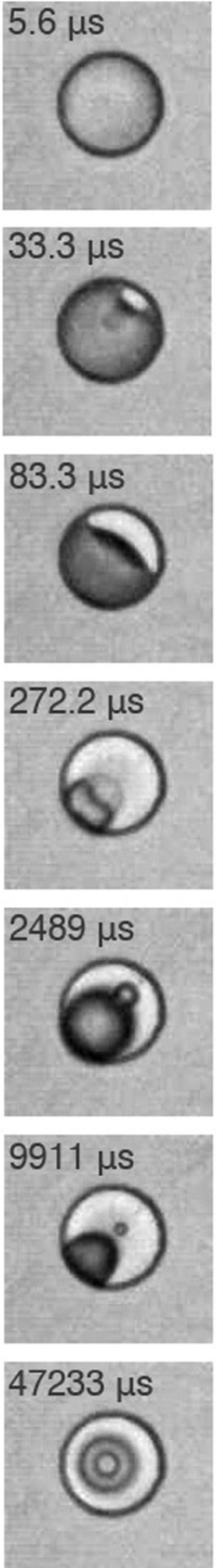
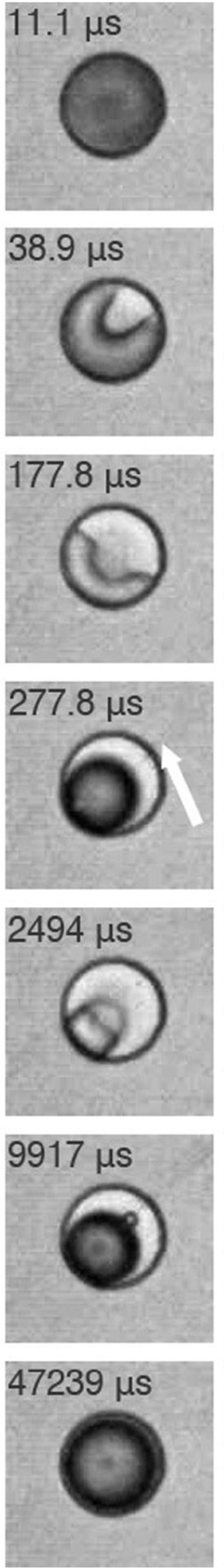
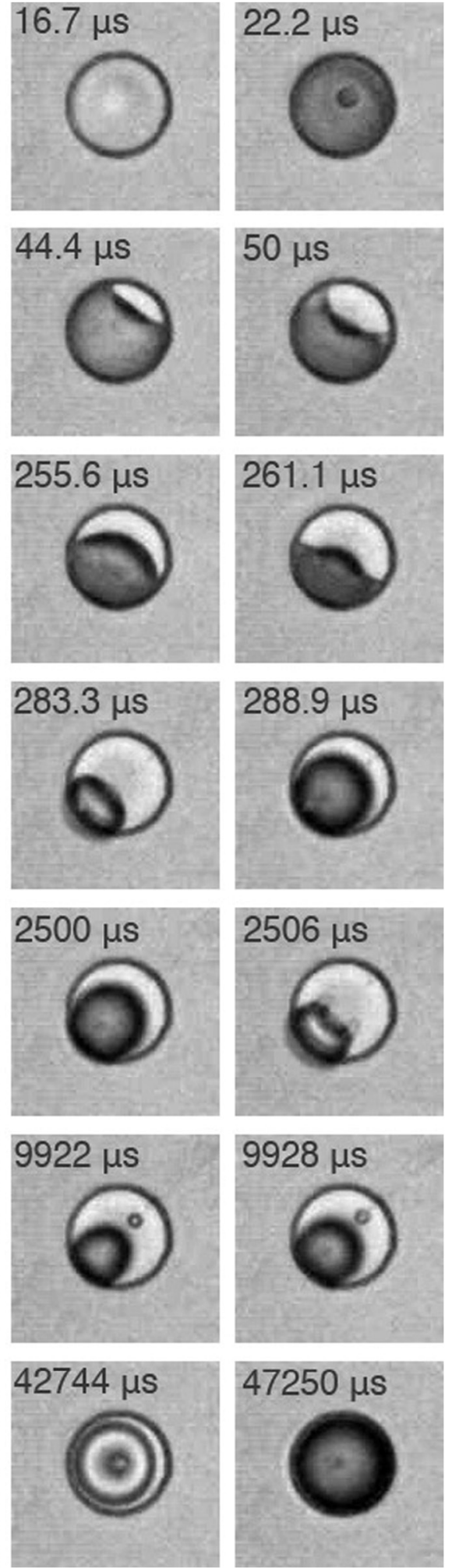

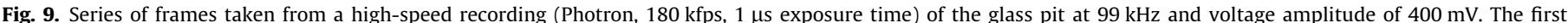

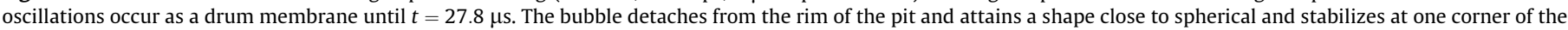

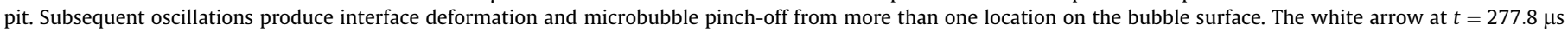
indicates a continuous stream of small bubbles ejected from the bubble interface along the new axis of symmetry formed by the pit and the bubble.

bubbles. For example, from the Rayleigh-Plesset equation [1] it follows that a spherical bubble with an equilibrium radius of $3 \mu \mathrm{m}$ would expand to a maximum radius of $4 \mu \mathrm{m}$ for an acoustic pressure amplitude of $90 \mathrm{kPa}$ at $80 \mathrm{kHz}$. Such (ejected) bubble expansions were not observed, therefore it follows that the local pressure value within the field of view must have been smaller than $90 \mathrm{kPa}$.

For the case of $99.5 \mathrm{kHz}$ (Section 3.2.1), a similar situation occurs as at $80 \mathrm{kHz}$. The stabilised and close-to-spherical bubble continuously emits smaller bubbles, but its size does not decrease 


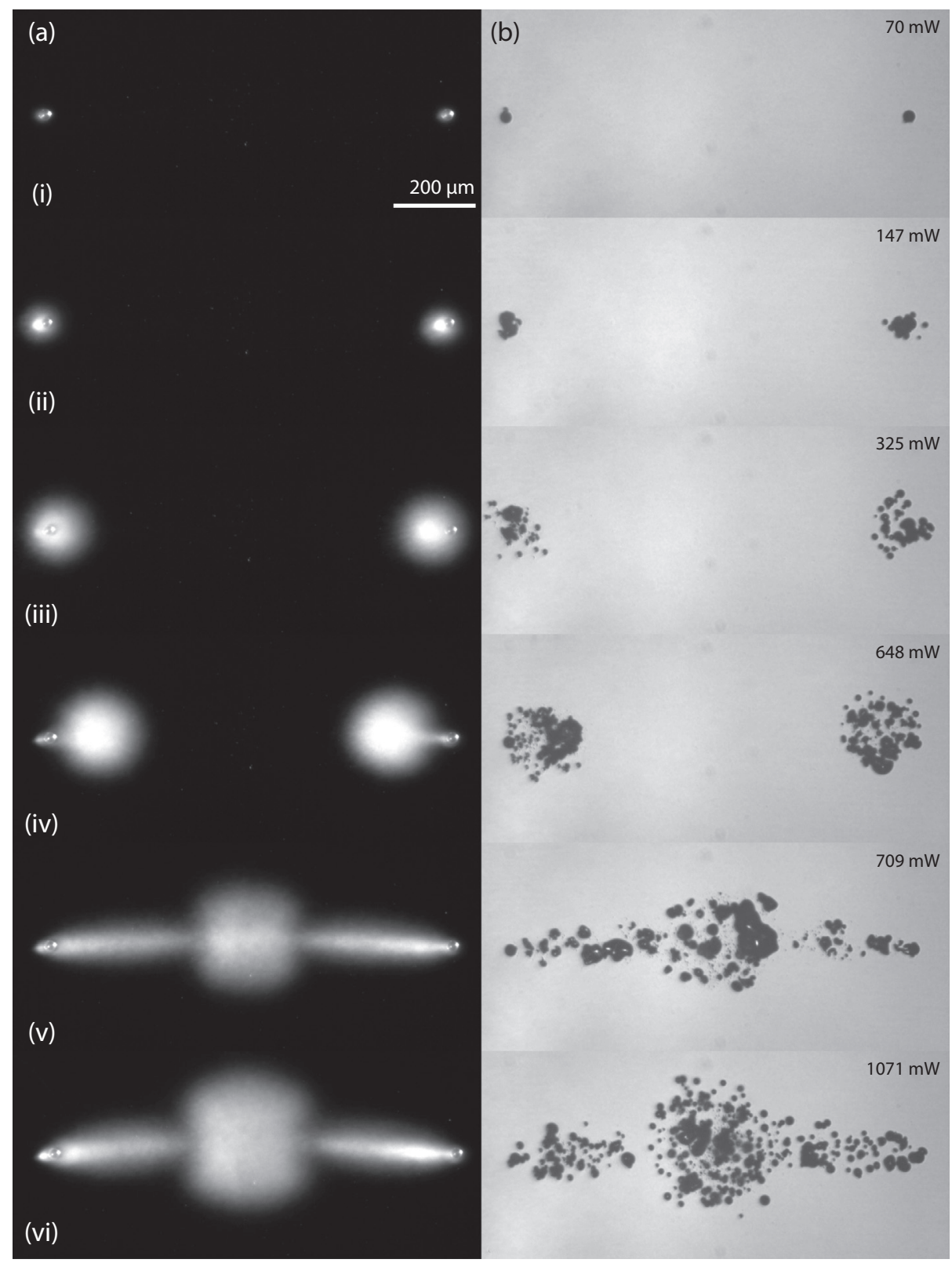

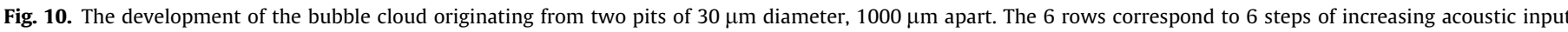

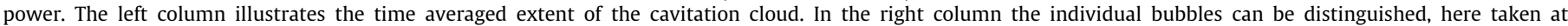
approximately maximum expansion.

nor does it dissolve all together. In this case the (horizontal) stream of bubbles ceased when the bubble detached from the lower left corner of the pit and relocated to the center of the pit $(t=47228 \mu \mathrm{s})$. Interestingly the bubble started to oscillate, alternating from spherical to ring or donut shaped (last row of Fig. 9).

Based on the observations made in the glass substrate (Section 3.1.2) we attribute the rings that appear on the interface to surface mode oscillations. From these images it can be inferred that the interface retains its axial symmetry and oscillates at the same frequency as the driving acoustic field. This symmetry is lost however when the bubble oscillations amplitude increase.

Remarkably, the pit bubble constantly ejected microbubbles while the loss of gas did not result in diminishing or deactivation of the process in all studied frequencies. The pinch-off rate corresponds to a gas volume flux in the order of $100 \mu \mathrm{m}^{3} / \mathrm{s}$ away from the pit, which must be balanced by an influx of equal magnitude. This transport must be of diffusive nature, where a rectifying mechanism can take place, similar to the well known process of rectified diffusion of spherical bubbles [44]. During oscillation of the meniscus, the gas pressure inside the pit bubble will rise during compression, and fall during expansion, respectively leading to gas diffusing out and into the pit bubble. Both the area effect and the shell effect may occur: the surface area of the meniscus is larger during expansion than during contraction, resulting in a net flow of gas into the pit bubble; the shell effect adds to this trend as the bubble expansion causes an increase of the dissolved gas concentration in the liquid adjacent to the meniscus while the opposite occurs during its contraction.

It is possible that the oscillation-induced microstreaming [45], draws in liquid from the bulk where the concentration of dissolved air is larger, thereby sustaining the pinch-off process. This same microstreaming can also account for the observed bubble trajectories, possibly in combination with primary and secondary Bjerkness forces [4]. 

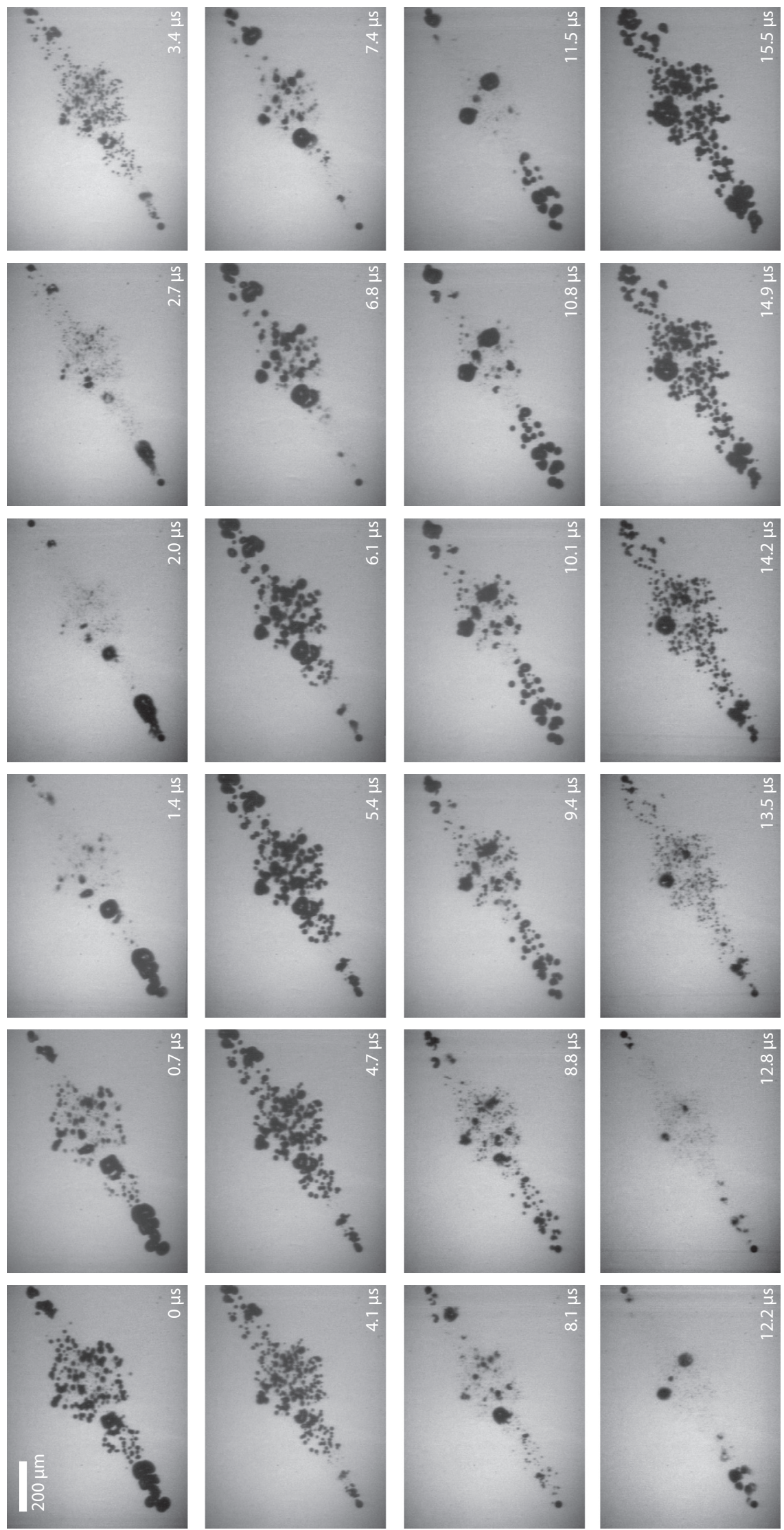

Fig. 11. Frames from a high-speed recording of the dual pit cavitation cloud at $810 \mathrm{~mW}$ input power and imaged at 1.4 Mfps. Supplementary Video. 


\section{Conclusion}

We have demonstrated that the introduction of artificially created crevice bubbles into an acoustically insonified liquid results in a myriad of acoustic cavitation related phenomena. Streams of microbubbles are ejected into the liquid while for larger input powers cavitation bubble clouds are formed. The stability of both the pit bubble and the cavitation bubble clouds is enabled by diffusion of gas back into the pit bubble. The results of the exploratory study described here confirms the common assumption that cavitation activity can be initiated and enhanced by the presence of crevice bubbles. However, the mechanism with which bubble release transpires is different from bubble detachment occurring in e.g. nucleate boiling and supersaturated liquids. In these heat and gas diffusion driven processes, the bubble displays unstable growth outside the perimeter of the pit after which it detaches due to buoyancy or drag forces. The inception of a cavitation bubble due to detachment from a pit bubble does not appear to involve such nucleation and subsequent detachment. Here, the released bubbles are typically smaller than the pit bubble and may result from large amplitude capillary waves which locally "fold" the liquid-gas interface into a singularities.

In this study three different frequencies were applied, each displaying distinct types of bubble generation and cavitation activity. For future studies a larger parameter space should be explored with additional focus on the effect of amplitude, pit dimensions and gas content in the liquid.

Clearly, the use of artificial crevice bubbles constitutes a new method (with advantages over conventional US horns or baths) to initiate and enhance cavitation at any desired location, provided the liquid and acoustic parameters are appropriate. The unique control over cavitation activity enables detailed investigation of acoustic cavitation and its effects such as shock wave emission, damage formation or sonochemistry, as was demonstrated in previous studies [10-12,39].

\section{Acknowledgements}

This study was funded by IMEC vzw. D.F.R research was supported by the Technology Foundation STW, Applied Science Division of NWO and the Technology Programme of the Ministry of Economic Affairs, The Netherlands. The authors would like to acknowledge the support in fabrication techniques provided by Stefan Schlautmann. The experiments in glass substrates were conducted at Nangyang University, Singapore with the support of Prof. Claus Dieter Ohl and Dr. Tandiono.

\section{Appendix A. Supplementary material}

Supplementary data associated with this article can be found, in the online version, at http://dx.doi.org/10.1016/j.ultras.2014.10. 002.

\section{References}

[1] C. Brennen, Cavitation and Bubble Dynamics, Oxford Engineering Science Series, Oxford University Press, 1995.

[2] K. Suslick, Y. Didenko, M. Fang, T. Hyeon, K. Kolbeck, W. McNamara, M. Mdleleni, M. Wong, Acoustic cavitation and its chemical consequences, Philos. Trans. R. Soc. London, Ser. A 357 (1751) (1999) 335.

[3] M. Ashokkumar, The characterization of acoustic cavitation bubbles: an overview, Ultrason. Sonochem. 18 (4) (2011) 864-872.

[4] L. Stricker, B. Dollet, D. Fernández Rivas, D. Lohse, Interacting bubble clouds and their sonochemical production, JASA 134 (1) (2013) 1854-1862.

[5] D. Fernandez Rivas, Taming Acoustic Cavitation, Ph.D. Thesis, University of Twente, 2012.

[6] D. Fernandez Rivas, P. Cintas, H.J.G.E. Gardeniers, Merging microfluidics and sonochemistry: towards greener and more efficient micro-sono-reactors, Chem. Commun. 48 (2012) 10935-10947.
[7] K. Yasuda, T. Torii, K. Yasui, Y. Iida, T. Tuziuti, M. Nakamura, Y. Asakura, Enhancement of sonochemical reaction of terephthalate ion by superposition of ultrasonic fields of various frequencies, Ultrason. Sonochem. 14 (6) (2007) 699-704.

[8] S. Muthukumaran, K. Yang, A. Seuren, S. Kentish, M. Ashokkumar, G.W. Stevens, F. Grieser, The use of ultrasonic cleaning for ultrafiltration membranes in the dairy industry, Sep. Purif. Technol. 39 (1-2) (2004) 99-107.

[9] T.J. Mason, L. Paniwnyk, J.P. Lorimer, The uses of ultrasound in food technology, Ultrason. Sonochem. 3 (3) (1996) S253-S260.

[10] D. Fernandez Rivas, A. Prosperetti, A.G. Zijlstra, D. Lohse, J.G.E. Gardeniers, Efficient sonochemistry through microbubbles generated with micromachined surfaces, Angew. Chem. Int. Ed. 49 (50) (2010) 9699-9701.

[11] D. Fernandez Rivas, L. Stricker, A.G. Zijlstra, H.J. Gardeniers, D. Lohse, A Prosperetti, Ultrasound artificially nucleated bubbles and their sonochemical radical production, Ultrason. Sonochem. 20 (1) (2013) 510-524.

[12] D. Fernandez Rivas, M. Ashokkumar, T. Leong, K. Yasui, T. Tuziuti, S. Kentish, D Lohse, J.G.E. Gardeniers, Sonoluminescence and sonochemiluminescence from a microreactor, Ultrason. Sonochem. 19 (6) (2012) 1252-1259.

[13] R.E. Apfel, Acoustic cavitation inception, Ultrasonics 22 (4) (1984) 167173.

[14] S. Helbig, S. Urban, E. Klein, S. Singh, Impact of megasonic process conditions on pre and sub-resolution assist feature damage, Photomask Technol. 7122 (1) (2008) 712210.

[15] W.J. Greenleaf, M.E. Bolander, G. Sarkar, M.B. Goldring, J.F. Greenleaf, Artificial cavitation nuclei significantly enhance acoustically induced cell transfection, Ultrasound Med. Biol. 24 (4) (1998) 587-595.

[16] D.M. Hallow, A.D. Mahajan, T.E. McCutchen, M.R. Prausnitz, Measurement and correlation of acoustic cavitation with cellular bioeffects, Ultrasound Med. Biol. 32 (7) (2006) 1111-1122.

[17] K. M, M. Goto, Size control of nanobubbles generated from shirasu-porousglass (spg) membranes, J. Membr. Sci. 281 (1-2) (2006) 386-396.

[18] B. Dollet, W. van Hoeve, J.P. Raven, P. Marmottant, M. Versluis, Role of the channel geometry on the bubble pinch-off in flow-focusing devices, Phys. Rev. Lett. 100 (3) (2008) 034504

[19] E.N. Harvey, D.K. Barnes, W.D. McElroy, A.H. Whiteley, D.C. Pease, K.W. Cooper Bubble formation in animals. i. Physical factors, J. Cell Comp. Physiol. 24 (1) (1944) 1-22.

[20] A.A. Atchley, A. Prosperetti, The crevice model of bubble nucleation, J. Acoust. Soc. Am. 86 (3) (1989) 1065-1084.

[21] S.F. Jones, G.M. Evans, K.P. Galvin, Bubble nucleation from gas cavities - a review, J. Coll. Interf. Sci. 80 (1) (1999) 27-50.

[22] G. Liger-Belair, M. Vignes-Adler, C. Voisin, B. Robillard, P. Jeandet, Kinetics of gas discharging in a glass of champagne: the role of nucleation sites, Langmuir 18 (4) (2002) 1294-1301.

[23] C.Y. Han, P. Griffith, The Mechanism of Heat Transfer in Nucleate Pool Boiling Tech. rep., Massachusetts Institute of Technology, Heat Transfer Laboratory, 1962.

[24] N. Bremond, M. Arora, C.D. Ohl, D. Lohse, Cavitation on surfaces, J. Phys.: Condens. Matter 17 (45) (2005) S3603.

[25] N. Bremond, M. Arora, C.D. Ohl, D. Lohse, Controlled multibubble surface cavitation, Phys. Rev. Lett. 96 (22) (2006) 224501.

[26] N. Bremond, M. Arora, S.M. Dammer, D. Lohse, Interaction of cavitation bubbles on a wall, Phys. Fluids 18 (12) (2006) 121505.

[27] B.M. Borkent, S. Gekle, A. Prosperetti, D. Lohse, Nucleation threshold and deactivation mechanisms of nanoscopic cavitation nuclei, Phys. Fluids 21 (10) (2009) 102003.

[28] C.H. Wang, V.K. Dhir, Effect of surface wettability on active nucleation site density during pool boiling of water on a vertical surface, J. Heat Transfer 115 (3) (1993) 659-669.

[29] D.E. Hughes, W.L. Nyborg, Cell disruption by ultrasound, Science 138 (3537) (1962) 108-114.

[30] S.-D. Howkins, Solid erosion in low-amplitude sound fields, J. Acoustical Soc. Am. 39 (1966) 55.

[31] T. Tandiono, D. Siak-Wei Ow, L. Driessen, C. Sze-Hui Chin, E. Klaseboer, A Boon-Hwa Choo, S.-W. Ohl, C.-D. Ohl, Sonolysis of escherichia coli and pichia pastoris in microfluidics, Lab Chip 12 (2012) 780-786.

[32] C.T. Chin, C. Lancée, J. Borsboom, F. Mastik, M.E. Frijlink, N. de Jong, M. Versluis, D. Lohse, Brandaris 128: a digital 25 million frames per second camera with 128 highly sensitive frames, Rev. Sci. Instr. 74 (12) (2003) 5026-5034.

[33] E.C. Gelderblom, H.J. Vos, F. Mastik, T. Faez, Y. Luan, T.J.A. Kokhuis, T.J.A. van der Steen, D. Lohse, N. de Jong, M. Versluis, Brandaris 128 ultra-high-speed imaging facility: 10 years of operation, updates, and enhanced features, Rev. Sci. Instrum. 83 (10) (2012) 103706.

[34] F.P. Schäfer, W. Schmidt, J. Volze, Organic dye solution laser, Appl. Phys. Lett. 9 (8) (1966) 306-309.

[35] A. van der Bos, A. Zijlstra, E.C. Gelderblom, M. Versluis, ilif: illumination by laser-induced fluorescence for single flash imaging on a nanoseconds timescale, Exp. Fluids 51 (2011) 1283-1289.

[36] H. Gelderblom, A. Zijlstra, L. van Wijngaarden, A. Prosperetti, Oscillations of a gas pocket on a liquid-covered solid surface, Phys. Fluids 12 (24) (2012) 122101.

[37] Tandiono, S. Ohl, D. Ow, E. Klaseboer, V. Wong, R. Dumke, C. Ohl, Sonochemistry and sonoluminescence in microfluidics, Proc. Natl. Acad. Sci. USA 108 (15) (2011) 5996-5998.

[38] D. Fernandez Rivas, B. Verhaagen, J.R.T. Seddon, A.G. Zijlstra, L.M. Jiang, L.W.M. van der Sluis, M. Versluis, D. Lohse, J.G.E. Gardeniers, Localized removal of 
layers of metal, polymer, or biomaterial by ultrasound cavitation bubbles, Biomicrofluidics 6 (3) (2012) 034114.

[39] D. Fernandez Rivas, J. Betjes, B. Verhaagen, W. Bouwhuis, T.C. Bor, D. Lohse J.G.E. Gardeniers, Erosion evolution in mono-crystalline silicon surfaces caused by acoustic cavitation bubbles, J. Appl. Phys. 113 (6) (2013) 064902.

[40] C.-D. Ohl, M. Arora, R. Ikink, N. de Jong, M. Versluis, M. Delius, D. Lohse, Sonoporation from jetting cavitation bubbles, Biophys. J. 91 (11) (2006) 4285 4295.

[41] W. van Hoeve, B. Dollet, M. Versluis, D. Lohse, Microbubble formation and pinch-off scaling exponent in flow-focusing devices, Phys. Fluids 23 (9) (2011) 092001.
[42] E. Castro-Hernandez, W. van Hoeve, D. Lohse, J.M. Gordillo, Microbubble generation in a co-flow device operated in a new regime, Lab Chip 11 (2011) 2023-2029.

[43] G.D. Crapper, An exact solution for progressive capillary waves of arbitrary amplitude, J. Fluid Mech. 2 (06) (1957) 532-540.

[44] A. Eller, H.G. Flynn, Rectified diffusion during nonlinear pulsations of cavitation bubbles, J. Acoust. Soc. Am. 37 (3) (1965) 493-503.

[45] D.L. Miller, Particle gathering and microstreaming near ultrasonically activated gas-filled micropores, J. Acoust. Soc. Am. 84 (4) (1988) 1378-1387. 